\title{
Internações Hospitalares por Neoplasias no Brasil, 2008-2018: Gastos e Tempo de Permanência
}

https://doi.org/10.32635/2176-9745.RBC.2020v66n3.992

Hospital Admissions for Neoplasms in Brazil, 2008-2018: Expenses and Lenght of Stay
Ingresos Hospitalarios por Neoplasias en Brasil, 2008-2018: Gastos y Duración de la Estadía

Hebert Luan Pereira Campos dos Santos'; Fernanda Beatriz Melo Maciel²; Rian Silva de Oliveira ${ }^{3}$

RESUMO

Introduçáo: As neoplasias representam a segunda causa de morte no Brasil, no entanto, garantir assistência plena e equilibrada ao diagnóstico e tratamento nas Regióes do país segue sendo um dos grandes desafios do sistema de saúde brasileiro. Objetivo: Descrever as taxas de internação por neoplasias no Brasil entre os anos de 2008 e 2018, mensurar os gastos hospitalares e o tempo de permanência das internaçôes por neoplasias no Sistema Único de Saúde (SUS). Método: Estudo ecológico de séries temporais com dados do Sistema de Informações Hospitalares do SUS. Resultados: Na série história de 2008-2018, houve 7.578.552 internaçôes por neoplasias no SUS, sendo $59 \%$ em pacientes do sexo feminino, $20 \%$ na faixa etária de 50 a 59 anos, $43 \%$ de cor branca e $44 \%$ na Região Sudeste do país. O gasto total das internaçóes hospitalares por neoplasias alcançou R \$ 13.225.554.102,73, com permanência média de 5,4 dias de internação por paciente. Conclusáo: Este é o primeiro estudo que utiliza como unidade territorial de análise todo o país; dessa maneira, espera-se que novos estudos envolvendo a morbidade hospitalar no país sejam desenvolvidos.

Palavras-chave: Neoplasias; Hospitalização/estatística \& dados numéricos; Custos Hospitalares/estatística \& dados numéricos; Gastos em Saúde/estatística \& dados numéricos; Epidemiologia Descritiva.

\section{ABSTRACT}

Introduction: Neoplasms represent the second leading cause of death in Brazil, however ensuring full and balanced assistance to diagnosis and treatment in the regions of the country remains one of the biggest challenges of the Brazilian health system. Objective: To describe the rates of hospitalization by neoplasms in Brazil between the years 2008 and 2018, to calculate hospital expenditures and the length of stay of hospitalizations by neoplasms in the Unified Health System (SUS). Method: Ecological study of time series with data from SUS Hospital Information System. Results: In the 2008-2018 history series, there were 7,578,552 hospitalizations for neoplasms in SUS, $59 \%$ of which in female patients, $20 \%$ in the age-range between 50 and 59 years old, $43 \%$, Caucasian and $44 \%$ in the country's southeastern region. The total cost of hospital admissions by neoplasms reached $\mathrm{R} \$ 13,225,554,102.73$, with an average stay of 5.4 days of hospitalization per patient. Conclusion: This is the first study that uses the entire country as a territorial unit of analysis, so it is expected that further studies involving hospital morbidity in the country will be developed.

Key words: Neoplasms; Hospitalization/statistics \& numerical data; Hospital Costs/statistics \& numerical data; Health Expenditures/statistics $\&$ numerical data; Epidemiology, Descriptive.

\section{RESUMEN}

Introducción: Las neoplasias representan la segunda causa de muerte en Brasil, sin embargo, garantizar una asistencia completa y equilibrada para el diagnóstico y el tratamiento en las regiones del país sigue siendo uno de los grandes desafíos del sistema de salud brasileño. Objetivo: Describir las tasas de hospitalización por neoplasias en Brasil entre los años 2008 y 2018, medir los gastos hospitalarios y la duración de la hospitalización por neoplasias en el Sistema Único de Salud (SUS). Método: Estudio ecológico de series de tiempo con datos del Información hospitalaria del SUS. Resultados: En la serie de historia 2008-2018, hubo 7.578 .552 hospitalizaciones por neoplasias en el SUS, el 59\% de los cuales eran pacientes de sexo femenino, el $20 \%$ tenían entre 50 y 59 años, $43 \%$ blancos y $44 \%$ en la región sureste del país. El costo total de los ingresos hospitalarios por neoplasias alcanzó R \$ 13.225.554.102,73, con una estadía promedio de 5,4 días de hospitalización por paciente. Conclusión: este es el primer estudio que utiliza todo el país como unidad de análisis territorial, por lo que se espera que se desarrollen más estudios sobre la morbilidad hospitalaria en el país. Palabras clave: Neoplasias; Hospitalización/estadística \& datos numéricos; Costos de Hospital/estadística \& datos numéricos; Gastos en Salud/estadística \& datos numéricos; Epidemiología Descriptiva.

\footnotetext{
${ }^{1}$ Instituto Multidisciplinar em Saúde (IMS)/Universidade Federal da Bahia (UFBA). Liga Acadêmica de Oncologia Clínica e Cirúrgica do Sudoeste Baiano. Vitória da Conquista (BA), Brasil. Orcid iD: https://orcid.org/0000-0003-2722-7945

2 UFBA. Vitória da Conquista (BA), Brasil. Orcid iD: https://orcid.org/0000-0002-6421-3940

${ }^{3}$ UFBA. Vitória da Conquista (BA), Brasil. Orcid iD: https://orcid.org/0000-0002-2162-132X

Endereço para correspondência: Hebert Luan Pereira Campos dos Santos. Rua Dário Ciacci, 705 - Candeias. Vitória da Conquista (BA), Brasil. CEP $45029-272$.

E-mail: ohebertluan@gmail.com
} 


\section{INTRODUÇÃO}

A morbimortalidade por câncer vem aumentando expressivamente em todo o mundo. Nos países em desenvolvimento tal fato decorre, em parte, pelo recente envelhecimento populacional, que gera crescimento exponencial de idosos. No Brasil, as neoplasias malignas representam a segunda causa de morte, no entanto, medidas estratégicas para o seu controle enfrentam problemas, demandando dos gestores do Sistema Único de Saúde (SUS) imensos esforços para garantir oferta adequada do cuidado em saúde ${ }^{1,2}$.

Os principais desafios para o sistema de saúde brasileiro consistem em garantir acesso pleno e equilibrado ao diagnóstico e ao tratamento da doença nas diferentes Regiōes do país ${ }^{2}$. Ao mesmo tempo, estudos reforçam a urgência de açóes operacionais estratégicas para reduzir a carga econômica do câncer nas economias em desenvolvimento ${ }^{3}$.

Cabe destacar que o custo econômico de um processo de adoecimento ou ocorrência em saúde envolvem custos diretos, indiretos e intangíveis. Os diretos abarcam custos médicos (exames, procedimentos, medicamentos, recursos humanos, consultas, internaçóes, reabilitação e outros) e açóes complementares, como as despesas de parentes e acompanhantes com transporte, dietas específicas, entre outros. Os indiretos referem-se aos gastos com perda de produtividade no mercado de trabalho e os intangíveis, por sua vez, às perdas em qualidade de vida ${ }^{4}$.

Nesse sentido, análises que permitam estimar os custos diretos médico-hospitalares tornam-se importantes para compreensão da carga da doença aos sistemas de saúde. Assim, o objetivo deste estudo foi descrever as taxas de internação por neoplasias no Brasil entre os anos de 2008 e 2018, mensurar os gastos hospitalares e o tempo de permanência das internaçôes por neoplasias no SUS.

\section{MÉTODO}

Foi realizado um estudo ecológico de série temporal com dados das internaçóes por neoplasias registradas no Sistema de Informaçôes Hospitalares (SIH) do SUS 5 , disponível no sítio eletrônico do Departamento de Informática do SUS (DATASUS). Os dados foram obtidos no mês de março de 2020. Foram selecionadas internaçóes por neoplasias ocorridas entre os anos de 2008 a 2018, registradas sob o código do Capítulo 2 da $10^{a}$ Revisão da Classificação Estatística Internacional de Doenças e Problemas Relacionados à Saúde (CID-10) .

O SIH/SUS é um banco de dados de domínio público e de acesso livre disponibilizado pelo Ministério da Saúde. Possibilita a obtençáo de dados referentes aos gastos hospitalares, contemplando unidades participantes do SUS, públicas e particulares conveniadas, apresentando parte dos custos diretos médico-hospitalares como valores das internaçôes, gastos referentes a serviços hospitalares e profissionais, dias de permanência, entre outros dados ${ }^{7}$.

Foi construída uma série histórica com números absolutos e calculadas as taxas de internaçáo segundo sexo (masculino; feminino), faixa etária (em anos: menor que 1,1 a $4 ; 5$ a $9 ; 10$ a $14 ; 15$ a $19 ; 20$ a 29; 30 a 39; 40 a 49; 50 a 59; 60 a 69; 70 a 79; 80 e mais), raça/cor (branco, preta, parda, amarela, indígena, sem informação) e Regiấo (Norte, Nordeste, Centro-Oeste, Sudeste, Sul) do país. Estimaram-se também os gastos do SUS com internaçôes hospitalares decorrentes das neoplasias (valores em Reais: gasto total, dos serviços hospitalares e dos serviços profissionais e gasto médio da internação), dias de permanência e permanência média em dias, todos referentes aos anos de 2008 a 2018.

Utilizou-se o software Microsoft Excel para Windows, versão 10, para as análises. Por utilizar exclusivamente dados anônimos e disponibilizados publicamente, conforme Portaria n. ${ }^{\circ}$ 466, de 2012, do Conselho Nacional de Saúde, não foi necessário submeter o projeto à apreciação de um Comitê de Ética em Pesquisa com Seres Humanos.

\section{RESULTADOS}

No período entre 2008 e 2018, no Brasil, foi registrado um total de 7.578 .552 internaçóes hospitalares por neoplasias. A série histórica desse período (Gráfico 1) permite observar que, ao longo dos anos, houve crescimento das internaçóes por câncer, porém, o ano de 2018 registrou uma queda não expressiva de 1,85\% referente ao ano anterior.

Ao estratificar esse contingente por sexo, verifica-se a predominância das internaçôes de pacientes do sexo feminino correspondendo a 59\%. Quando analisado com

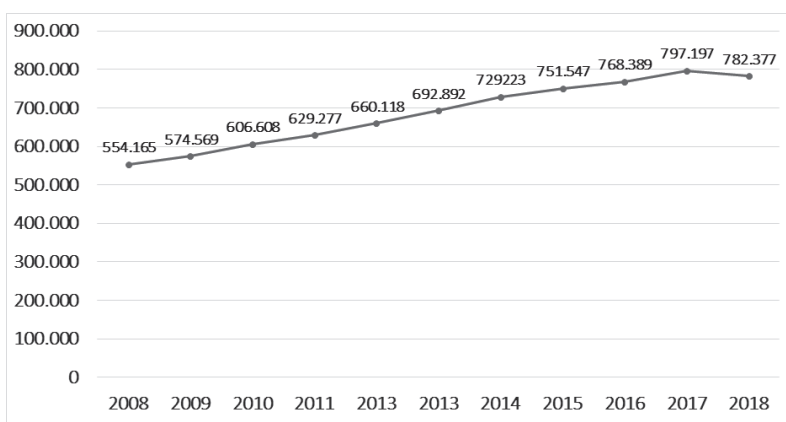

Gráfico 1. Internações hospitalares por neoplasias no Brasil entre os anos de 2008 e 2018

Fonte: SIH/SUS 5 . 
base na faixa etária, observa-se que, do total de internaçôes, $57,4 \%$ ocorreram em pessoas acima de 50 anos de idade. No quesito raça/cor, há predominância de indivíduos de cor branca (43\%), no entanto, cabe destacar que 22\% dos pacientes tiveram esse campo não preenchido. A distribuição geográfica das internações hospitalares por neoplasias, com base nas Regióes do território brasileiro, revela que $44 \%$ das internaçóes registradas no SUS se concentraram no Sudeste do país (Tabela 1).

Referente aos custos diretos médico-hospitalares, os gastos totais com internações hospitalares, decorrentes de neoplasias entre 2008 e 2018, no âmbito do SUS, foram de

Tabela 1. Número absoluto e percentual de internação por neoplasias segundo sexo, faixa etária, raça/cor e Região, Brasil, 2008-2018

\begin{tabular}{|c|c|c|}
\hline Variáveis & $\mathbf{N}$ & $\%$ \\
\hline \multicolumn{3}{|l|}{ Sexo } \\
\hline Masculino & 3.111 .126 & 41,00 \\
\hline Feminino & 4.467 .426 & 59,00 \\
\hline \multicolumn{3}{|c|}{ Faixa etária (em anos) } \\
\hline Menor de 1 ano & 26.719 & 0,35 \\
\hline 1-4 anos & 143.882 & 2,00 \\
\hline $5-9$ anos & 139.061 & 1,83 \\
\hline 10-14 anos & 145.502 & 1,92 \\
\hline 15-19 anos & 168.748 & 2,22 \\
\hline 20-29 anos & 378.502 & 5,00 \\
\hline $30-39$ anos & 758.943 & 10,01 \\
\hline 40-49 anos & 1.433 .838 & 18,92 \\
\hline $50-59$ anos & 1.517 .240 & 20,02 \\
\hline $60-69$ anos & 1.476 .677 & 19,48 \\
\hline 70-79 anos & 985.842 & 13,01 \\
\hline 80 anos e mais & 403.598 & 5,32 \\
\hline \multicolumn{3}{|l|}{ Raça/cor } \\
\hline Branca & 3.244 .583 & 42,81 \\
\hline Preta & 306.371 & 4,04 \\
\hline Parda & 2.303 .100 & 30,38 \\
\hline Amarela & 75.241 & 1,00 \\
\hline Indígena & 4.973 & 0,06 \\
\hline Sem informação & 1.643 .984 & 21,69 \\
\hline \multicolumn{3}{|l|}{ Região } \\
\hline Norte & 314.151 & 4,14 \\
\hline Nordeste & 1.841 .841 & 24,30 \\
\hline Sudeste & 3.334 .196 & 44,00 \\
\hline Sul & 1.605 .049 & 21,18 \\
\hline Centro-Oeste & 483.315 & 6,37 \\
\hline Total & 7.578 .552 & 100 \\
\hline
\end{tabular}

Fonte: SIH/SUS ${ }^{5}$.
$\mathrm{R} \$ 13.225 .554 .102,73$, dos quais, $\mathrm{R} \$ 10.359 .637 .600,61$ corresponderam a gastos com serviços hospitalares e $\mathrm{R} \$$ 2.807.793.325,14 com serviços profissionais. O valor médio de internação por neoplasias nesse período foi de $\mathrm{R} \$ 1.745,13$. No que diz respeito ao tempo de internação, foram contabilizados, no total, 41.216.608 dias de permanência nos hospitais, com uma permanência média de 5,4 dias de internação por paciente (Tabela 2).

\section{DISCUSSÃO}

Verificou-se, após os resultados do estudo, que a magnitude das internaçóes por neoplasias entre os anos de 2008 e 2018 foram crescentes, porém com tendência estável de crescimento. Dados divulgados pelo Instituto Nacional de Câncer José Alencar Gomes da Silva (INCA) ${ }^{1}$ revelam que a doença está entre as quatro principais causas de morte prematura (antes dos 70 anos de idade) em diversos países, e a previsão, para cada ano do triênio 2020-2022, é que ocorram cerca de 625 mil novos casos de câncer no país ${ }^{1}$.

Cabe destacar que o Brasil passa por um processo de transição tanto demográfica como epidemiológica, sendo o aumento do envelhecimento populacional um dos maiores desafios para a saúde pública contemporânea ${ }^{8}$. Ao mesmo tempo, os custos socioeconômicos associados às doenças crônicas não transmissíveis, nas quais estão inseridas as neoplasias, acarretam repercussóes na economia dos países, sendo estimado gasto de US\$ 7 trilhōes, durante 2011-2025, em países de baixa e média rendas. Segundo o Instituto Brasileiro de Geografia e Estatística (IBGE) ${ }^{10}$, em 2030, o Brasil terá mais idosos do que crianças e jovens, e a mortalidade por câncer terá crescido $45 \%$.

Os dados apresentados por este estudo, referentes ao predomínio de internaçôes por câncer em mulheres, corroboram o que é apresentado em literatura, uma vez que em países em desenvolvimento, como o Brasil, é esperado o predomínio de câncer no sexo feminino, em razão dos altos índices de detecção de neoplasias específicas a esse gênero, como o câncer do colo uterino ${ }^{11-13}$. Deve-se considerar ainda que a maior concentração de internação de pacientes do sexo feminino também pode ser reflexo da população feminina ser maior em determinadas Regiôes demográficas ${ }^{14}$. Estudos apontam também que mulheres buscam e usam mais os serviços, tanto em consultas quanto em internaçóes, o que pode estar relacionado com a detecçáo precoce da doença e a maior chance de internação em tempo oportuno para o cuidado ${ }^{15}$.

No que tange ao maior número de internaçóes entre pessoas maiores de 50 anos de idade, este estudo corrobora os dados encontrados na literatura ${ }^{4,14,16}$. Sousa-Muñoz et. al. ${ }^{12}$ pontuam o aumento progressivo da incidência de 
Tabela 2. Gastos (em Reais), tempo e média de permanência (em dias) das internações por neoplasias segundo Região do país, Brasil, $2008-2018$

\begin{tabular}{ccccccc}
\hline Regiáo & $\begin{array}{c}\text { Valor total do gasto } \\
\text { (RS) }\end{array}$ & $\begin{array}{c}\text { Valor dos serviços } \\
\text { hospitalares (RS) }\end{array}$ & $\begin{array}{c}\text { Valor dos serviços } \\
\text { profissionais (RS) }\end{array}$ & $\begin{array}{c}\text { Valor médio por } \\
\text { internação (RS) }\end{array}$ & $\begin{array}{c}\text { Permanência } \\
\text { total (em dias) }\end{array}$ & $\begin{array}{c}\text { Média de } \\
\text { permanência } \\
\text { (em dias) }\end{array}$ \\
\hline Norte & $395.707 .683,18$ & $301.533 .533,58$ & $91.802 .180,34$ & $1.259,61$ & 2.131 .201 & 6,8 \\
Nordeste & $3.527 .351 .432,65$ & $2.684 .610 .451,33$ & $817.720 .678,77$ & $1.915,12$ & 9.178 .049 & 5,0 \\
Sudeste & $5.804 .752 .007,09$ & $4.608 .893 .264,74$ & $1.175 .458 .269,25$ & $1.740,98$ & 18.916 .422 & 5,7 \\
Sul & $2.771 .422 .067,06$ & $2.204 .838 .170,60$ & $560.105 .088,86$ & $1.726,69$ & 8.477 .604 & 5,3 \\
Centro-Oeste & $726.320 .912,75$ & $559.762 .180,36$ & $162.707 .107,92$ & $1.502,79$ & 2.513 .332 & 5,2 \\
\hline Total & $13.225 .554 .102,73$ & $10.359 .637 .600,61$ & $1.745,13$ & $2.807 .793 .325,14$ & 41.216 .608 & 5,4 \\
\hline
\end{tabular}

Fonte: SIH/SUS 5 .

câncer com o avanço da idade e como isso implica em diferentes necessidades de cuidados de saúde, sobretudo, no que tange às internaçôes hospitalares. Em geral, pessoas idosas tendem a consumir mais os serviços de saúde, sendo as taxas de internaçáa e o tempo de ocupaçáo do leito por esse grupo populacional mais elevado do que o de indivíduos de outras faixas etárias ${ }^{13,14,16}$.

Sousa-Muñoz et al. ${ }^{12}$ apresentam ainda um dado consoante ao verificado neste estudo que é a menor frequência de internamentos por câncer em idosos acima de 80 anos, o que pode estar relacionado ao fato de se tratar de uma doença com alta letalidade e de duraçáo, em geral, relativamente curta. Deve-se destacar ainda que a idade é um marcador de risco importante e definidor do prognóstico do paciente.

Alguns estudos ${ }^{17,18}$ apontam que, ao comparar a incidência de câncer em adultos e crianças ( 0 a 19 anos), este último grupo apresenta menor ocorrência. No Brasil, corresponde a cerca $3 \%$ de todos os tumores malignos ${ }^{18}$, corroborando os dados encontrados de menores indicadores de internaçáo por essa causa nessa faixa etária.

Quanto à variável raça/cor, constatou-se que quase metade das internaçôes ocorre em pessoas consideradas brancas. O que chama atenção neste trabalho é a porcentagem de pacientes que tiverem essa informação não preenchida, esse dado converge com uma análise realizada das internações hospitalares por neoplasias no Estado do Rio Grande do Sul, que encontrou uma porcentagem de $14,71 \%{ }^{19}$. Em seu estudo, Farias et al. ${ }^{20}$ chamaram atenção para o fato de que todas as variáveis no $\mathrm{SIH} /$ SUS apresentavam $100 \%$ de completude, no entanto, a variável raça/cor está bastante aquém dos demais sistemas de informação em saúde brasileiro, tais quais: o Sistema de Informação sobre Mortalidade (SIM) e o Sistema de Informaçôes sobre Nascidos Vivos (Sinasc).

A existência de desigualdades étnicas em saúde no país torna o não preenchimento desse dado um fator que impacta diretamente nas análises das iniquidades (injustas e evitáveis) em saúde e dificulta a elaboração de políticas públicas mais equânimes ${ }^{21}$. Vale pontuar também que a indisponibilidade ou incompletude de informações individualizadas por parte dos sistemas de informação em saúde do país dificultam o desenvolvimento de estudos epidemiológicos a partir de dados secundários.

Um estudo que buscou investigar a associação entre raça/cor e a sobrevivência em uma coorte de mulheres com câncer de mama evidenciou que mulheres negras foram diagnosticadas em estadiamentos mais avançados e possuíram menor sobrevivência em comparação com as brancas $^{22}$. Outro trabalho brasileiro de base populacional demonstrou que mulheres brancas possuíam maior acesso à mamografia de rastreamento ${ }^{23}$. Apesar das limitaçóes metodológicas desses estudos como obstáculos para os estudos epidemiológicos de raça e saúde ${ }^{21}$, é convincente que o preenchimento e a leitura dessa categoria se fazem extremamente importantes.

Neste estudo, a Região Sudeste apresentou o maior número de internaçóes hospitalares decorrentes de neoplasias. Os dados encontrados relacionam-se diretamente com o que foi projetado pelo INCA para cada ano do triênio 2020-2022, no qual a Regiáo Sudeste concentra mais de $60 \%$ da incidência. Deve-se considerar que a distribuição por tipo de câncer varia entre as macrorregióes brasileiras e, mesmo que exista um declínio de casos de neoplasias associados a condiçóes socioeconômicas desfavoráveis, em algumas Regiôes do Brasil, esse perfil persiste. Torna-se um desafio ainda maior para o sistema de saúde brasileiro o desenvolvimento de políticas públicas que deem conta de equacionar as desigualdades tanto socioeconômicas quanto de acesso aos serviços de saúde pela população ${ }^{1}$.

Alguns autores ${ }^{24,25}$ mostram que a assistência médica e a distribuição de leitos no país encontram-se concentradas no Sudeste, sendo que a Regiáo Sul apresenta a maior 
média de leitos por mil habitantes do país. Um estudo divulgado pelo Observatório de Oncologia demonstrou que, em 2015, o câncer se configurava como a principal causa de morte em 516 municípios brasileiros, porém a maioria desses municípios encontra-se nas Regiôes mais desenvolvidas do país, onde a expectativa de vida e o Índice de Desenvolvimento Humano (IDH) são maiores. Oitenta por cento desses municípios ficam localizados nas Regiôes Sul e Sudeste do país, as quais possuem maior número de serviços de saúde disponíveis, portanto maior opção de diagnóstico, e com populaçôes mais idosas, o que resulta em maiores prevalências de câncer ${ }^{26}$.

Referente aos gastos hospitalares por neoplasias pelo SUS, foram encontrados poucos estudos ${ }^{27,28}$ que trouxessem essa estimativa, tendo como unidade geográfica de análise o Brasil. Considerando esta série histórica de 10 anos, no período de 2008 a 2018, o custo foi de R $\$$ 13,2 bilhóes, com o maior percentual correspondendo aos gastos relativos aos serviços hospitalares. $\mathrm{O}$ valor médio da internação foi de $\mathrm{R} \$ 1.745,13$, porém existiram diferenças importantes entre as Regiōes. Outros autores já apontavam diferenças nos recursos empregados pelo SUS com internaçôes hospitalares nas diferentes macrorregióes brasileiras, sendo maiores nas Regiōes Sul e Sudeste e menor na Região Norte ${ }^{1,29}$. Os dados apresentados aqui demonstram que, para as internaçóes por neoplasias, as Regiōes Sudeste e Nordeste possuem os maiores custos; e a Regiăo Norte apresenta os menores custos despendidos.

O valor médio referente à internação estima o gasto médio de recursos despendidos pelo SUS na prestação de atendimento hospitalar, porém é influenciado por fatores socioeconômicos, epidemiológicos e demográficos que determinam o perfil da demanda hospitalar do SUS. Apresenta uma limitação importante para série histórica, já que os valores pagos são apresentados em Reais correntes do ano e não incluem abonos, fatores de reposição, incentivos e complementaçóes estaduais e municipais. Salienta-se que os dados dos custos apresentados não devem ser utilizados de modo isolado, mas sim auxiliares na elaboração de estratégias para reavaliar políticas públicas de saúde e estimular a política de negociação de preços e remuneração da tabela ${ }^{30,31}$.

A média nacional de permanência foi de 5,4 dias, sendo que a Regiāo Norte apresentou a média de permanência mais elevada, próxima a uma semana. Esse indicador avalia em média o tempo que o paciente permanece internado no hospital e pode auxiliar na avaliação da eficiência da gestão operacional e rotatividade dos leitos, bem como nas práticas clínicas adotadas. Vale ressaltar que diversos fatores influenciam tanto no tempo de permanência como nos gastos hospitalares decorrentes das neoplasias, tais quais: tipo e estadiamento do câncer, idade, presença ou não de comorbidades, agilidade e disponibilidade para realização e resultados de exames, entre outros. Acrescenta-se que o uso isolado da média de tempo de internamento e o volume elevado de gastos não fornecem, em si, informaçôes suficientes para sugerir um bom ou mau uso do recurso ${ }^{31,32}$.

As limitaçôes do presente estudo ancoram-se na compreensão de que o SIH/SUS é um banco de dados de cunho administrativo, o qual se organiza a partir da lógica de pagamento da atenção hospitalar. É um sistema que não possui caráter universal, isto é, registra apenas as internaçôes realizadas no âmbito do sistema público de saúde, podendo existir dupla ou tripla contagem de um mesmo paciente, em razão de o sistema não identificar reinternaçôes e transferências de outros hospitais. Somam-se a isto a cobertura irregular no preenchimento dos dados e a confiabilidade das informaçóes entre as Regiōes do país, além das diferenças regionais na cobertura dos serviços privados de internação, os quais privilegiam a Regiáo Sudeste, dificultando a comparabilidade entre essas Regiōes?

Outras limitaçóes importantes deste estudo compreendem a falta de uma análise estatística robusta e a não realização de uma padronização por idade, uma vez que dificultam a comparabilidade das taxas de internaçóes entre diferentes Regióes, quando apresentam estruturas etárias distintas, além da possibilidade de os gastos estarem subestimados, já que existe financiamento complementar ao valor da tabela do sistema. Quanto aos gastos, evidenciase também que foram analisados apenas os custos diretos médico- -hospitalares, deixando de fora os custos diretos de açôes complementares, custos indiretos, ajustes monetários referentes à inflação e a descontos, custos intangíveis, os quais impactam diretamente a economia em saúde, o indivíduo, as famílias e a sociedade ${ }^{7,33}$.

Apesar das barreiras, assinala-se que o SIH/SUS representa cerca de $70 \%$ das internaçóes realizadas no país e é um dos sistemas mais utilizados pelos diversos níveis de gestão dos serviços de saúde ${ }^{7}$, embora não abranja, no contexto do tratamento oncológico, as modalidades terapêuticas de radioterapia e quimioterapia, as quais são, majoritariamente, realizadas ambulatorialmente, sujeitas à liberaçáo da Autorizaçáo de Procedimento Ambulatorial de Alta Complexidade (Apac) ${ }^{34}$.

Os custos e a complexidade operacional envolvidos no desenvolvimento de estudos epidemiológicos de base populacional tornam-se empecilhos para sua ampla utilização; nesse sentido, os dados secundários gerados pelos sistemas nacionais de informação em saúde são uma boa alternativa, devendo seu uso ser estimulado pela diversidade de conteúdos e complexidade de análises possíveis, considerando as limitaçôes apresentadas ${ }^{35}$. 


\section{CONCLUSÃO}

Apesar das limitações do presente estudo, pode-se concluir que as internaçôes por neoplasias no SUS durante o período estudado predominaram em indivíduos do sexo feminino, acima dos 50 anos de idade, residentes na Região Sudeste do Brasil onde se concentrou os maiores gastos em internaçôes por neoplasias. Tais achados são importantes para pensar estratégias de planejamento operacional das açóes de saúde e acesso aos serviços, já que revelam diferenças regionais importantes, as quais podem ser reflexo de um processo histórico de organizaçáo da atençáo à saúde no país. Espera-se que este estudo estimule o desenvolvimento de estudos com análises estatísticas robustas envolvendo custo ou custo-efetividade das internaçôes por neoplasias no país.

\section{CONTRIBUIÇÕES}

Todos os autores contribuíram substancialmente na concepção ou no planejamento do estudo; na obtenção, na análise e/ou interpretação dos dados e na redação e/ou revisão crítica e aprovaram a versão final a ser publicada.

\section{DECLARAÇÃO DE CONFLITO DE INTERESSES}

Nada a declarar.

\section{FONTES DE FINANCIAMENTO}

Não há.

\section{REFERÊNCIAS}

1. Instituto Nacional de Câncer José Alencar Gomes da Silva. Estimativa 2020: incidência de câncer no Brasil [Internet]. Rio de Janeiro: INCA; 2019 [acesso 2020 mar 7]. Disponível em: https://www.inca.gov.br/sites/ ufu.sti.inca.local/files/media/document/estimativa-2020incidencia-de-cancer-no-brasil.pdf

2. Oliveira EXG, Melo ECP, Pinheiro RS, et al. Acesso à assistência oncológica: mapeamento dos fluxos origem-destino das internaçóes e dos atendimentos ambulatoriais. O caso do câncer de mama. Cad Saúde Pública. 2011;27(2):317-326. doi: http://doi. org/10.1590/S0102-311X2011000200013

3. Cancela MC, Almeida LM de. Impacto econômico da mortalidade prematura por câncer nos Brics. Rede Câncer [Internet]. 2018 mar;40:32-33 [acesso 2020 jun 6]. Disponível em: https://www.inca.gov.br/sites/ufu.sti. inca.local/files//media/document/rrc-40-artigo-impactoeconomico-da-mortalidade-prematura-por-cancer-nosbrics_0.pdf

4. Silva EN, Silva MT, Pereira MG. Identificação, mensuração e valoração de custos em saúde. Epidemiol
Serv Saúde. 2016;25(2):437-439. doi: http://doi. org/10.5123/S1679-49742016000200023

5. SIHD: Sistema de Informaçóes Hospitalares Descentralizado [Internet]. Rio de Janeiro: Sistema de Informaçóes Hospitalares. C2016 [acesso 2020 mar 12]. Disponível em: http://sihd.datasus.gov.br/principal/ index.php

6. Ministério da Saúde (BR), Departamento de Informática do Sistema Único de Saúde [Internet]. Brasília, DF: DATASUS; [data desconhecida]. Morbidade Hospitalar do SUS CID-10: lista de tabulaçáo para morbidade; [1989] [acesso 2020 mar 12]. Disponível em http:// tabnet.datasus.gov.br/cgi/sih/mxcid10lm.htm

7. Bittencourt SA, Camacho LAB, Leal MC. O sistema de informaçáo hospitalar e sua aplicação na saúde coletiva. Cad Saúde Pública. 2006;22(1):19-30. doi: https://doi. org/10.1590/S0102-311X2006000100003

8. Duca GFD, Nader GA, Santos IS, et al. Hospitalização e fatores associados entre residentes de instituiçóes de longa permanência para idosos. Cad Saúde Pública. 2010;26(7):1403-10. doi: https://doi.org/10.1590/ S0102-311X2010000700019

9. Malta DC, Bernal RTI, Lima MG, et al. Doenças crônicas não transmissíveis e a utilização de serviços de saúde: análise da Pesquisa Nacional de Saúde no Brasil. Rev Saúde Pública. 2017;51(Supl. 1):4s. doi: https://doi. org/10.1590/s1518-8787.2017051000090

10. Instituto Brasileiro de Geografia e Estatística [Internet]. Rio de Janeiro: IBGE; c2020. Censo 2010; 2010 [acesso 2020 abr 2]. Disponível em: http://www.censo2010. ibge.gov.br/

11. Souza RS, Simão DAS, Lima EDRP. Perfil sociodemográfico e clínico de pacientes atendidos em um serviço ambulatorial de quimioterapia paliativa em Belo Horizonte. REME Rev Min Enferm [Internet]. 2012 [acesso 2020 mar 14];16(1):38-47. Disponível em: http://www.reme.org.br/artigo/detalhes/498

12. Sousa-Muñoz RL, Formiga MYQ, Silva AEVF, et al. Hospitalizaçôes por neoplasias em idosos no âmbito do Sistema Único de Saúde na Paraíba, Brasil. Rev Saúde Pesq. 2015;8(3):479-91. doi: https://doi. org/10.17765/2176-9206.2015v8n3p479-491

13. Santos MAS, Oliveira MM, Andrade SSCA, et al. Tendências da morbidade hospitalar por doenças crônicas não transmissíveis no Brasil, 2002 a 2012. Epidemiol Serv Saúde. 2015;24(3):389-98. doi: https://doi.org/10.5123/ S1679-49742015000300005

14. Levorato CD, Mello LM, Silva AS, et al. Fatores associados à procura por serviços de saúde numa perspectiva relacional de gênero. Ciênc Saúde Coletiva. 2014;19(4):1263-74. doi: https://doi.org/10.1590/141381232014194.01242013

15. Ciałkowska-Rysz A, Kowalczyk M, Gottwald L, et al. The comparison of common cancer types and the coincidence of concomitant chronic diseases between palliative home care patients in Lodz Voivodeship and the general Polish 
population. Arch Med Sci. 2012;8(3):496-503. doi: https://doi.org/10.5114/aoms.2012.29406

16. Oliveira MM, Malta DC, Guauche H, et al. Estimativa de pessoas com diagnóstico de câncer no Brasil: dados da pesquisa nacional de saúde, 2013. Rev Bras Epidemiol. 2015;18(Supl 2):146-57. doi: https://doi. org/10.1590/1980-5497201500060013

17. Pan R, Marques AR, Costa Júnior ML, et al. Caracterização das internaçóes hospitalares de crianças e adolescentes com neoplasias. Rev Latino-Am Enferm. 2011;19(6):1413-20. doi: https://doi.org/10.1590/ S0104-11692011000600019

18. Instituto Nacional de Câncer José Alencar Gomes da Silva. Incidência, mortalidade e morbidade hospitalar por câncer em crianças, adolescentes e adultos jovens no Brasil: informaçóes dos registros de câncer e do sistema de mortalidade [Internet]. Rio de Janeiro: INCA; 2016 [acesso 2020 mar 6]. Disponível em: https://www.inca.gov.br/ sites/ufu.sti.inca.local/files//media/document//incidenciamortalidade-morbidade-hospitalar-por-cancer.pdf

19. Dazzi MC, Zatti CA, Baldissera R. Internaçôes hospitalares por neoplasias no estado do Rio Grande do Sul. Braz J Surg Clin Res [Internet]. 2014 [acesso 2020 mar 20];7(2):05-09. Disponível em: https://www. mastereditora.com.br/periodico/20140702_165043.pdf

20. Farias YN, Leite IC, Siqueira MAMT, et al. Iniquidades étnico-raciais nas hospitalizaçóes por causas evitáveis em menores de cinco anos no Brasil, 2009-2014. Cad Saúde Pública. 2019;35(Supl 3):e00001019. doi: https://doi. org/10.1590/0102-311x00001019

21. Chor D. Desigualdades em saúde no Brasil: é preciso ter raça [correspondência]. Cad Saúde Pública. 2013;29(7):1272-75. doi: https://doi.org/10.1590/ S0102-311X2013000700002

22. Nogueira MC, Guerra MR, Cintra JRD, et al. Disparidade racial na sobrevivência em 10 anos para o câncer de mama: uma análise de mediação usando abordagem de respostas potenciais. Cad Saúde Pública. 2018;34(9):e00211717. doi: https://doi. org/10.1590/0102-311x00211717

23. Leal MC, Gama SGN, Frias P, et al. Healthy lifestyles and access to periodic health exams among Brazilian women. Cad Saúde Pública. 2005;21(Suppl 1):S78-S88. doi: https://doi.org/10.1590/S0102311X2005000700009

24. Meneghell SN, Armani TB, Rosa RS, et al. Internaçôes hospitalares no Rio Grande do Sul. Inf Epidemiol Sus. 1997;6(2):49-59. doi: https://doi.org/10.5123/S010416731997000200006

25. Panis C, Kawasaki ACB, Pascotto CR, et al. Revisão crítica da mortalidade por câncer usando registros hospitalares e anos potenciais de vida perdidos. Einstein. 2018;16(1):1-7. doi: https://doi.org/10.1590/s167945082018ao4018

26. Observatório de Oncologia [Internet]. São Paulo: ABRALE; [data desconhecida]. Cepas T. Câncer como a primeira causa de morte nos municípios brasileiros; 2018 abr 18 [acesso 2020 mar 2]. Disponível em: https://observatoriodeoncologia.com.br/cancer-comoa-primeira-causa-de-morte-nos-municipios-brasileirosem-2015/

27. Siqueira ASE, Gonçalves JG, Mendonça PEX, et al. Economic impact analysis of cancer in the health system of Brazil: model based in public database. Health Sci J. 2017;11(4):514. doi: https://doi.org/10.21767/1791809X.1000514

28. Pearce A, Sharp L, Hanly P, et al. Productivity losses due to premature mortality from cancer in Brazil, Russia, India, China, and South Africa (BRICS): a populationbased comparison. Cancer Epidemiol. 2018;53:27-34. doi: https://doi.org/10.1016/j.canep.2017.12.013

29. Peixoto SV, Giatti L, Afradique ME, et al. Custo das internaçóes hospitalares entre idosos brasileiros no âmbito do Sistema Único de Saúde. Epidemiol Serv Saúde. 2004;13(4):239-46. doi: https://doi.org/10.5123/ S1679-49742004000400006

30. Ministério da Saúde (BR). Valor médio pago por internação hospitalar no SUS - E.11 [Internet]. [2005] [acesso 2020 mar 20]. Disponível em: http://tabnet. datasus.gov.br/tabdata/LivroIDB/2edrev/e11.pdf.

31. Knust RE, Portela MC, Pereira CCA, et al. Estimated costs of advanced lung cancer care in a public reference hospital. Rev Saúde Pública. 2017;51:53. doi: https:// doi.org/10.1590/s1518-8787.2017051006665.

32. Agência Nacional de Saúde Suplementar. Média de permanência geral [Internet]. V1.02. Rio de Janeiro: ANS; 2013 [acesso 2020 mar 29]. Disponível em: http:// www.ans.gov.br/images/stories/prestadores/E-EFI-05.pdf

33. Drumond EF, Machado CJ, Vasconcelos MR, et al. Utilização de dados secundários do SIM, Sinasc e SIH na produçáo científica brasileira de 1990 a 2006. Rev Bras Estud Popul. 2009;26(1):7-19. doi: https://doi. org/10.1590/S0102-30982009000100002

34. Ministério da Saúde (BR). Manual de bases técnicas da oncologia SIA/SUS - sistema de informações ambulatoriais. 25. ed. Brasília, DF: Ministério da Saúde; 2019 maio [acesso 2020 mar 26]. Disponível em: https:// www.inca.gov.br/sites/ufu.sti.inca.local/files//media/ document/manual-oncologia-25a-edicao_0.pdf

35. Castro VC, Borghi AC, Mariano PP, et al. Perfil de internaçôes hospitalares de idosos no âmbito do Sistema Único de Saúde. Rev Rene [Internet]. 2013 [acesso 2020 abr 6];14(4):791-800. Disponível em: http://www. periodicos.ufc.br/rene/article/view/3547

Recebido em 16/4/2020 Aprovado em 19/6/2020 